\title{
Habitat choice by marine zooplankton in a high-latitude ecosystem
}

\author{
Maria Fossheim ${ }^{1,2, *}$, Raul Primicerio ${ }^{1}$ \\ ${ }^{1}$ Norwegian College of Fishery Science, University of Tromsø, 9037 Tromsø, Norway \\ ${ }^{2}$ Present address: Institute of Marine Research Tromsø, PO Box 6404, 9294 Tromsø, Norway
}

\begin{abstract}
According to habitat choice theory, zooplanktonic prey should choose a depth habitat that maximizes food intake while minimizing predation risk. Body size affects competitive ability and vulnerability in zooplankton, thereby influencing the adaptive choice of depth habitat. To study sizedependent habitat choice in marine zooplankton, we sampled their vertical distributions on a finescale with an optical plankton counter (OPC) during a post-bloom condition in late spring on the continental shelf off North Norway. Taxonomic information was obtained from net samples. We found that small and large zooplankton segregated along the water column under resource heterogeneity, with large zooplankton aggregating in the resource-rich habitat together with predators of small zooplankton, in accordance with multi-trophic level habitat choice theory. These patterns of habitat use differ from summer vertical distributions documented in Norwegian waters, when the distribution of small and large zooplankton is reversed. To account for the discrepancy, we propose a new habitat choice model that considers the different predation regimes experienced in spring vs. summer.
\end{abstract}

KEY WORDS: Habitat selection · Competitive displacement · Size-selective predation · Predator avoidance $\cdot$ Habitat refuge

Resale or republication not permitted without written consent of the publisher

\section{INTRODUCTION}

The marine pelagic environment is spatially heterogeneous with respect to resources and predators of zooplankton (Pinel-Alloul 1995). Resource availability and predation risk are often correlated, producing a food and safety axis of heterogeneity, where highresource habitats are riskier (Brown 1998). Along the water column, sharp vertical gradients of food availability and predation risk occur on a spatial scale that allows an active choice of habitat by zooplankton. The individual zooplankter may therefore optimize foraging by balancing the resource availability and predation risk of visited patches (Leibold \& Tessier 1997). Risk sensitive behaviour by zooplankton might then lead to a negative spatial correlation between zooplankton and their food (Folt \& Burns 1999).

In the absence of predators, individual foragers are expected to distribute themselves so as to optimize net energy intake, using food patches according to an ideal free distribution (IFD), resulting in resource matching (Fretwell \& Lucas 1969). In the presence of predators, prey (foragers) tend to redistribute themselves depending on the hunting habitat of their predators. Habitat choice theory predicts that predators of zooplankton should hunt in the habitat nutritionally most profitable for their prey (Sih 1998, Lima 2002), which for planktonic herbivores is most often near the surface. Zooplankton prey should, however, be distributed more uniformly among patches (Sih 1998). If resource-poor patches offer a refuge from predation, prey aggregation tends to decrease in the resource-rich patches (i.e. undermatching).

Different groups of foragers (e.g. species or size groups) are rarely equally vulnerable to predation and are expected to evolve different habitat choices. Typically, resistant prey, which invest in anti-predator defences, have higher resource requirements and therefore tend to use resource-rich habitats. On the other hand, more vulnerable prey have lower resource 
demands and can thrive in resource-poor habitats. Due to lower resource requirements, the vulnerable prey will be competitively superior to the resistant prey (Brown 1998). Accordingly, the vulnerable, but competitively superior prey, is expected to use the nutritionally poor habitat refuge, most often located in the deeper part of the water column, to avoid predation, whereas the resistant prey remains in the food-rich habitat with the predator (Brown 1998, Primicerio 2005). Unequal prey competitors are thereby expected to partition the water column, segregating in different depth habitats.

Body size affects competitive ability and vulnerability of zooplankton, influencing the adaptive choice of depth habitat (Woodward et al. 2005). In particular, zooplankton prey size will determine vulnerability in the presence of size-selective predators. Predators with limited mouth gaps, such as chaetognaths and juvenile fish, feed more efficiently on smaller zooplankton prey (Persson et al. 1996, Baier \& Purcell 1997). Different zooplankton size groups will also differ in competitive ability. More specifically, zooplankton with larger body sizes attain zero intrinsic growth rates on higher critical food concentrations than smaller zooplankton (Hirst \& Bunker 2003), making the latter group a superior competitor when resources become limited (Tokeshi 1999). The most efficient species or stage reduces the resource of a habitat to a level that is unfavourable for less efficient species or stages (Brown 1998), making small zooplankton able to competitively displace larger zooplankton from their chosen habitat. In the presence of predators feeding in a resource-rich habitat, small zooplankton species will choose resource-poor habitats, whereas large zoo- plankton will overlap with predators (Adler et al. 2001).

Habitat use consistent with a choice based on foraging and anti-predator behaviour has been shown in a model of krill and penguin behaviour in the Antarctic ocean (Alonzo et al. 2003) and has been generalized in a model for many different marine fish and benthic communities (Dill et al. 2003). Habitat refuge use has even been shown to cause trophic cascades in marine benthic communities (Grabowski \& Kimbro 2005). Adaptive habitat use is also well documented in lakes (Leibold \& Tessier 1997). The above foraging adaptations are likely to be as important in the marine pelagic as elsewhere (Dill et al. 2003), but the lack of documentation may be partly due to sampling challenges set by a strongly advective environment.

The present study provides an extensive coverage of zooplankton vertical distribution, with fine resolution data obtained with an optical plankton counter (OPC) during a post-bloom condition in late spring on the continental shelf off North Norway (Fig. 1). This extensive sampling protocol allows us to study the repeatability of distributional patterns over a large area, an empirical objective that is not obtainable with traditional net-based sampling. The study presents sizestructured zooplankton data that enable us to illustrate and test to what extent habitat choice may be operating among planktonic consumers in a marine community. In this high-latitude ecosystem the patterns of habitat use involve only few species and are not complicated by diel vertical migration, since the study is performed during midnight sun (Blachowiak-Samolyk et al. 2006), simplifying the test of habitat choice hypotheses.

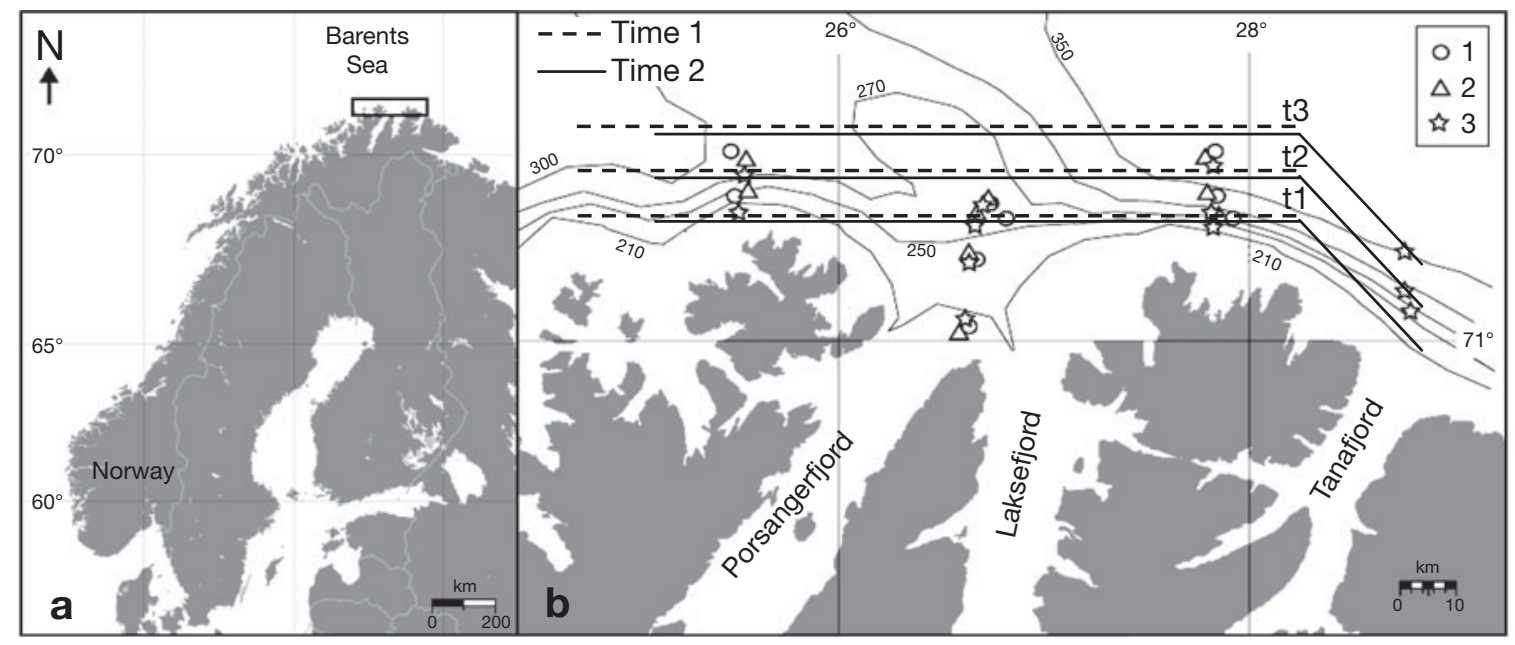

Fig. 1. (a) Location of study area in the southern Barents Sea off the coast of Norway. (b) ScanFish transect lines (t1, t2 and $\mathrm{t} 3$ ) for the 2 sampling periods (Time 1: May 21-23; Time 2: May 28-30) in 2001. MOCNESS stations May 18-21 (O); May 23-24 ( $\Delta$ ); May 26-27 (战) 
Our objective was to document adaptive depth habitat use by marine zooplankton, particularly with regard to multi-trophic level habitat choice theory and the use of habitat refuges. Focusing on a 3-trophic level food chain with a gape-limited predator with a preference for small zooplankton, we hypothesized that: (1) where the water column is heterogeneous with regard to resources, small zooplankton prey will undermatch resources and stay mostly in the habitat refuge, whereas large zooplankton prey will overmatch resources; and (2) where the water column is homogeneous with regard to resources, zooplankton prey (large and small) will be distributed uniformly throughout the water column.

\section{MATERIALS AND METHODS}

Sampling protocol. The field sampling was undertaken in May 2001 in the southern Barents Sea (Fig. 1). Sampling of hydrography and zooplankton throughout the water column was performed using an undulating platform (ScanFish MKII, EIVA-GMI), repeatedly towed from the surface down to $100 \mathrm{~m}$ depth along transects during 2 sampling periods (Time 1: May 21 to 23; Time 2: May 28 to 30; Fig. 1), each lasting about 48 h. An OPC (Focal Technologies) and a CTD (SeaBird 911+, Sea-Bird Electronics) with a Seapoint chlorophyll fluorometer (Seapoint Sensors) were mounted on the ScanFish. The fluorometer is an in vivo fluorescence sensor that gives relative values of chlorophyll $a$ $\left(\mathrm{mg} \mathrm{m}^{-3}\right)$, scaled from 0 to 10 .

OPC specification. The OPC automatically counts and measures the size of particles in the size range 0.25 to $14 \mathrm{~mm}$ equivalent spherical diameter (ESD), targeting meso-zooplankton to krill. The OPC continuously counts and measures all particles that pass through the sampling tunnel and reports back to the computer every half second $(2 \mathrm{~Hz})$. The possible resolution of the OPC is thus dependent on the speed of the towing vehicle, in our case the ScanFish, deployed vertically $100 \mathrm{~m}$ in approx. $10 \mathrm{~min}$. Our OPC thus reports for every $8 \mathrm{~cm}$ of the water column that is sampled. In this study the zooplankton distribution was recorded in 878 water columns over an area of ca. $116 \times 19 \mathrm{~km}$. Hence, the OPC provides size-structured zooplankton data at a spatial and temporal resolution largely superior to net sampling.

An in-depth outline of the OPC is given in Herman (1992). Considerable effort has been put into studying the OPC's performance in measuring zooplankton. The general conclusion when comparing with net samples is that the OPC can be used to describe zooplankton communities (Heath et al. 1999). Since the OPC deals with particles rather than species, it may be difficult to tell whether counts originate from zooplankton or nonliving material, but it has been shown that the OPC is capable of making reliable measurements on zooplankton even at high detritus concentrations (Zhang et al. 2000). Another concern has been whether particles originating from phytoplankton (single cells or aggregates) are being counted by the OPC. The OPC used in our study was tested under post-bloom conditions in May to June 1998 in a high-latitude fjord ecosystem with many of the same features found offshelf, in our study area, by Edvardsen et al. (2002). The authors of that study observed negative correlations of counts versus fluorescence and concluded that phytoplankton aggregates were very unlikely to contaminate the zooplankton measurements made by the OPC.

OPC data processing and analyses. Integrated OPC size spectrum data of 40 classes of equal $\log _{10}$ ESD were extracted and used for the analyses. The data for each water column profile (considered 1 sample), from the 3 transects and 2 time periods (Fig. 1), were integrated over $2 \mathrm{~m}$ depth intervals from 5 to $95 \mathrm{~m}$ depth. This integration was necessary to obtain statistically manageable data without too many zero recordings, especially for less abundant size classes (i.e. larger zooplankton). The data set was cut off at each end (surface and bottom) to avoid error recordings due to instrument behaviour (e.g. the ScanFish angle and speed changes when turning). Since the OPC may sample the water column slightly differently in the upward versus downward direction, the results presented are only from downward movements of the OPC. The other half of the data set, from the upward movements of the OPC, was also analysed, giving similar results.

To test the habitat choice hypotheses using OPC data, Pearson correlation coefficients between fluorescence and abundance of small and large zooplankton were calculated to estimate the degree of matching between consumer and resource distributions. The $95 \%$ CI of the correlation coefficients were calculated by bootstrapping to avoid biased estimates (Davison \& Hinkley 1997), using the program R. The correlation coefficients were ordered according to maximum fluorescence in each profile, with maximum fluorescence being used as a proxy for the degree of resource heterogeneity throughout the water column. Maximum fluorescence has the advantage of incorporating both the level of blooming and the level of heterogeneity since only 2 patterns of fluorescence distribution throughout the water column were observed: one where the resource level was low in the whole water column (homogeneous columns); and another with a high resource level in one part of the water column (heterogeneous columns). Low maximum fluorescence 
values were thus from water columns with vertically homogeneous resource levels, whereas high maximum fluorescence values were from water columns with heterogeneous resource levels.

Taxonomic classification of size groups. Before and between the ScanFish sampling periods, net samples of the water column were obtained by MOCNESS at 30 stations (Fig. 1). The zooplankton community found in the net samples is briefly summarized in Fig. 2, and has been presented previously by Fossheim et al. (2006). The coupled sampling by MOCNESS and OPC, and the low diversity of high-latitude pelagic communities, allows us to reconstruct community structure from counts of different size classes. Species present at more than $5 \%$ of the total abundance in the MOCNESS samples were assigned to 4 different size groups: $0.25-$ $1.00,1.00-1.40,1.40-2.00$ and 2.00-14 mm ESD, hereafter referred to as small, medium, large and xlarge zooplankton size groups, respectively (Fig. 2). The size grouping was based on laboratory experiments running monocultures of live zooplankton species through the OPC. Zooplankton taxa for which OPC size signatures were unavailable were assigned to a size group based on literature values of prosome lengths and body volumes, except for Oikopleura spp., which was estimated to be within a similar size range as that of Mallotus villosus larvae (based on personal observation of live Oikopleura spp. and M. villosus larvae from the nets).

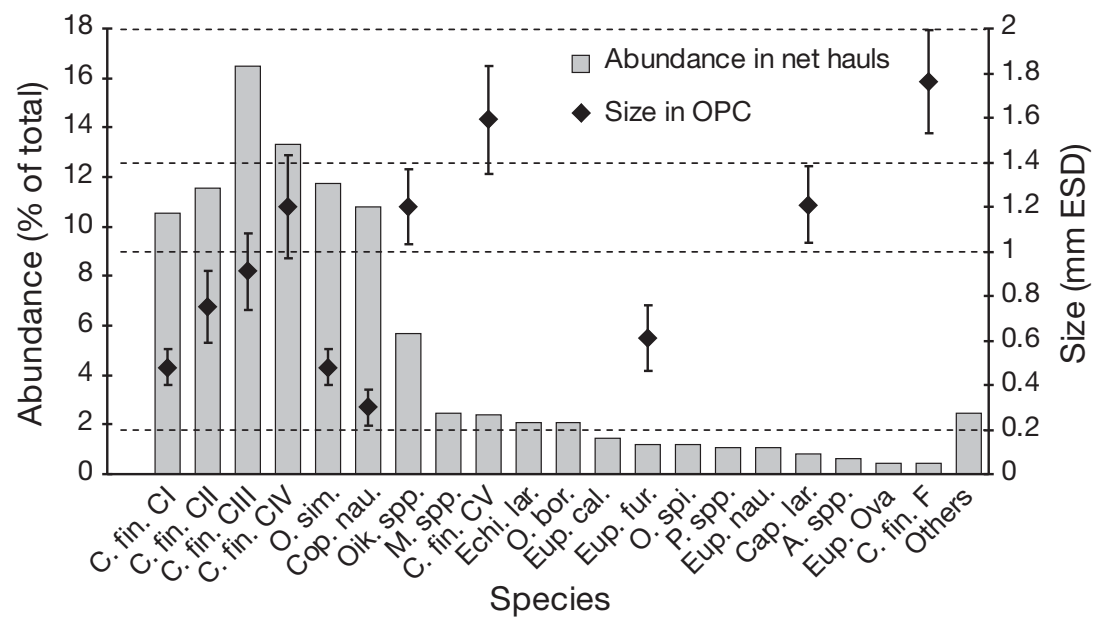

Fig. 2. Relative abundance (\% of total, bars) of zooplankton species in the MOCNESS (left $y$-axis) and their sizes (mean \pm SD, diamonds and whiskers) in $\mathrm{mm}$ equivalent spherical diameter (ESD) measured by the optical plankton counter (OPC; right $y$-axis). Horizontal dotted lines show the size group intervals: small, 0.25-1.00; medium, 1.00-1.40; large, 1.40-2.00 mm ESD. C. fin.: Calanus finmarchicus; O. sim.: Oithona similes; Cop. nau.: copepod nauplii; Oik. spp.: Oikopleura spp.; M. spp.: Metridia spp.; Echi. lar: echinoderm larva; O. bor.: Oncaea borealis; Eup. (cal., fur., ova, nau.): euphausids (calyptopis, furcilia, ova, nauplii); O. spi.: Oithona spinirostris; P. spp.: Pseudocalanus spp.; Cap. lar.: capelin larvae (Mallotus villosus); A. spp.: Acartia spp.
The small zooplankton size group was expected to include mostly Calanus finmarchicus copepodite stages I through III (CI through CIII), but also copepod nauplii (Mauchline 1998) and Oithona similis (Ashjian et al. 2003). The medium zooplankton size group was expected to include C. finmarchicus CIV and Oikopleura spp. (as well as Mallotus villosus larvae in small proportions). The size group large zooplankton was expected to include $C$. finmarchicus $\mathrm{CV}$ and females (Fig. 2). According to the net samples, possible contributors to the size group xlarge zooplankton $(>2 \mathrm{~mm}$ included chaetognaths (e.g. Sagitta elegans and (Medusae spp. and Aglanspp.) and the amphipod Themisto abyssorum (these small zooplankton (Baier \& Purcell 1997, 2005)

MOCNESS data analyses. The more extensive sampling by ScanFish relative to the MOCNESS limits direct comparison of the distributional patterns found the data obtained with the 2 sampling devices. Most portantly, the 30 MOCNESS stations were not repby the ScanFish (range 0 to 6.6), but covered a fluorescence range from 0 to 2.8. For fluorescen higher than 3 at the ScanFish stations, heterogeneity in the water column was observed. When testing habitat choice hypotheses that assume resource heterogeneity throughout the water column, our MOCNESS data were therefore less suitable than the OPC data. However, an exploratory analysis of the MOCNESS data was nevertheless useful, since some degree of resource heterogeneity in the water column was indicated at 9 of the 30 stations (max. fluorescence $>1$ ), which allows us to check the distribution of known predators on small zooplankton. At these stations we compared the fluorescence distribution with the distribution of the identified xlarge zooplankton (i.e. the predators: chaetognaths, jellyfish and the amphipod Themisto abyssorum) and the distribution of the most abundant small zooplankter, the herbivore Calanus finmarchicus CIII (Fig. 2). According to habitat choice theory the predator (xlarge zooplankton) should match the resource (of their prey), and the prey (C. finmarchicus CIII) should undermatch the resource (i.e. use the habitat refuge). 


\section{RESULTS}

\section{Patterns in OPC data}

The environment in the study area was clearly heterogeneous with respect to resource availability, both horizontally and vertically, as can be seen in the fluorescence data in Fig. 3 (fluorescence panel). The transect in Fig. 3 includes areas with homogeneous and heterogeneous water columns with regard to fluorescence (i.e. resource availability) in both coastal and oceanic water masses (see Fig. 4 in Fossheim et al. 2005). However, no relationship between the vertical distribution of fluorescence and physical conditions was found, either with respect to temperature or salinity, or in relation to different water masses. Explanations of the vertical patterns of fluorescence based on oceanographic conditions (e.g. fronts, eddies etc.) are therefore not discussed any further in this paper, but see Fossheim et al. (2005) for a discussion on the observed horizontal patterns.

The distribution of zooplankton abundance obtained with the OPC along the 3 transects was highly patchy both horizontally and vertically. The distribution of the 4 size groups differed, as shown in transect $\mathrm{t} 3$, Time 2 (Fig. 3). Horizontally, the degree of patchiness increased with increasing zooplankton size. Vertically, the degree of patchiness varied depending on fluorescence level, with high fluorescence values associated with high patchiness in all zooplankton sizes. With increasing zooplankton size, overall abundance decreased. There were no indications of diel variation in depth distribution of zooplankton abundance for any size group.

Small zooplankton were systematically absent from fluorescence maxima (Fig. 3, S panel), whereas medium zooplankton were found on the steep gradient of the fluorescence patch (Fig. 3, M panel). The abundance of large zooplankton matched fluorescence maxima irrespective of depth (Fig. 3, L panel), as did the xlarge zooplankton (Fig. 3, XL panel). Large and xlarge zooplankton were nearly absent from low fluorescence areas. The observed patterns were consistent across time and space, independent of differing physical settings (Fossheim et al. 2005), and the same patterns in all size groups were repeated in several water column replicates from different water masses.

In heterogeneous environments (max. fluorescence > $3)$, where fluorescence varied throughout the water column, small zooplankton undermatched resources, being negatively correlated with fluorescence, whereas large zooplankton overmatched resources (Fig. 4). Hence, small zooplankton were not found in resource-rich
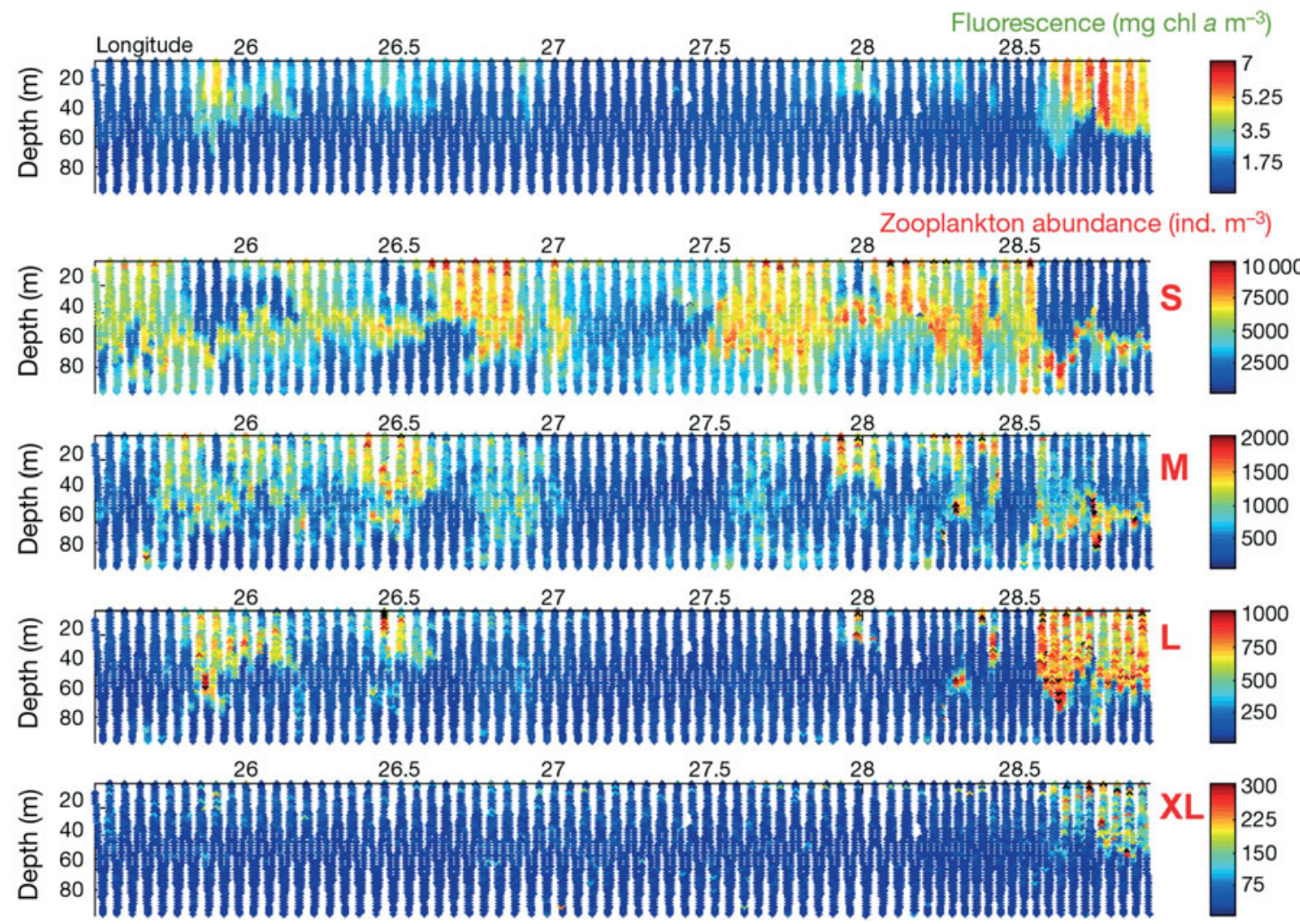

Fig. 3. Fluorescence and abundance of small, medium, large and extra large zooplankton (S: 0.25-1.00, M: 1.00-1.40, L: 1.40-2.00 and XL: 2.00-14 mm ESD, respectively) in transect t3, Time 2 (see Fig. 1) 

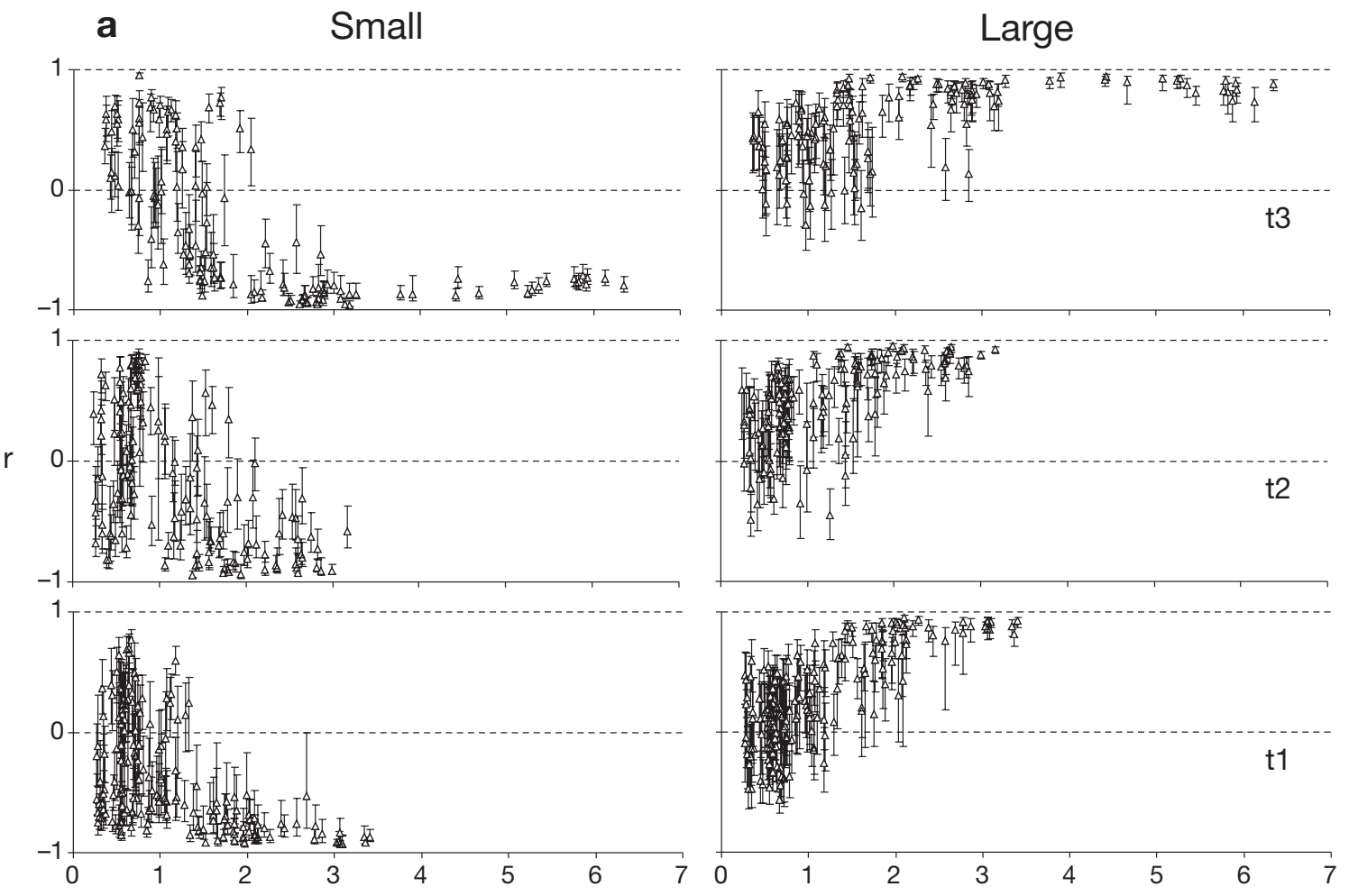

b
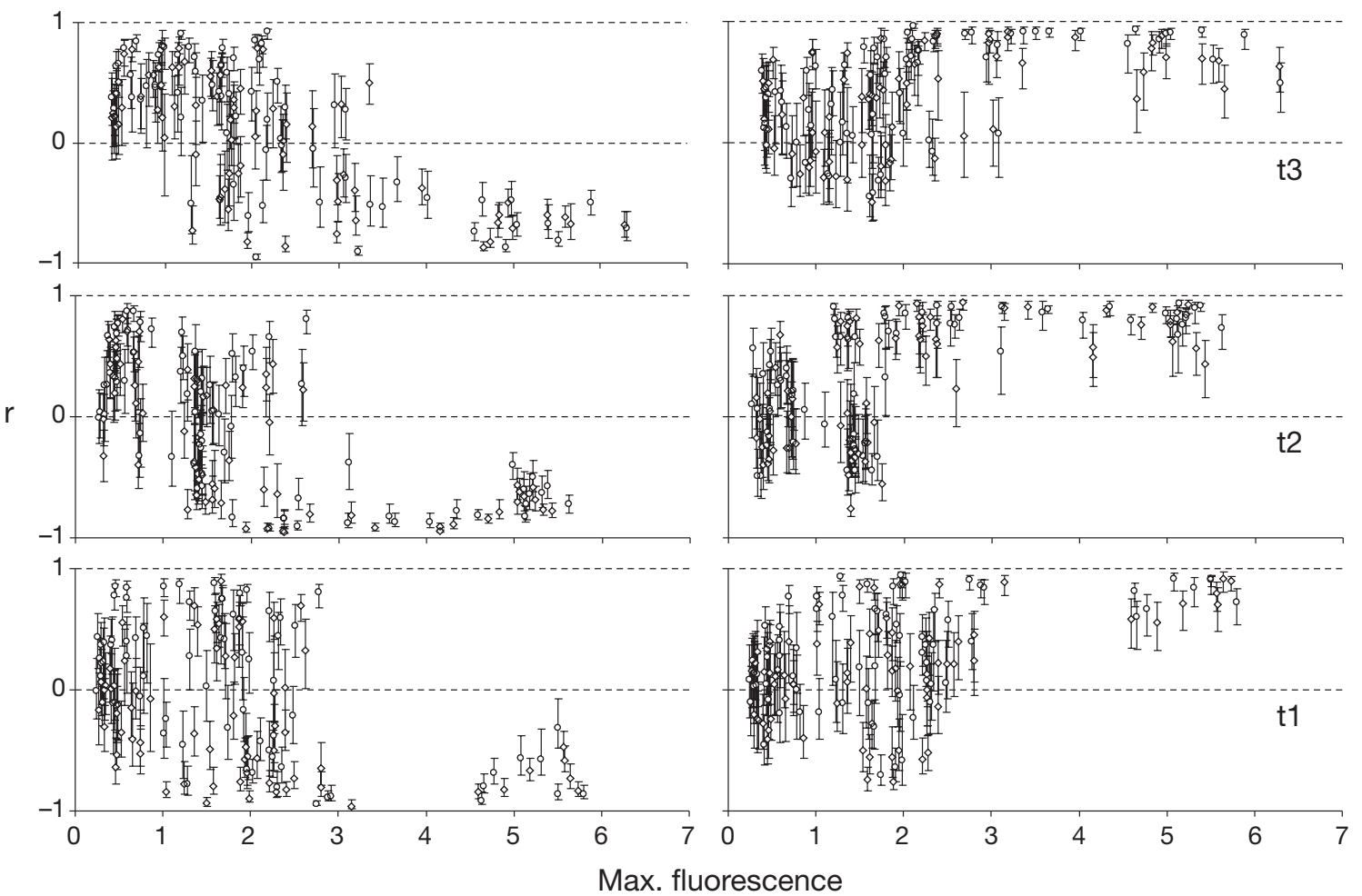

Fig. 4. Pearson correlation coefficients (whiskers, bootstrapped 95\% CI) between fluorescence and abundance of zooplankton (small and large). A positive correlation implies resource matching by zooplankton (i.e. the distributions of fluorescence and zooplankton along the water column overlap), whereas a negative correlation implies undermatching (i.e. the distributions of fluorescence and zooplankton do not overlap). Each water column profile is arranged according to maximum fluorescence in each profile for transects t1, t2 and $\mathrm{t} 3$ (see Fig. 1) for the 2 sampling periods in 2001: (a) Time 1, May 21-23; (b) Time 2, May 28-30 
patches together with large zooplankton. In homogeneous environments (max. fluorescence $<2$ ), where fluorescence did not vary throughout the water column, small and large zooplankton either matched resources, i.e. were positively correlated with fluorescence, or were uncorrelated with fluorescence (Fig. 4). Hence, small and large zooplankton were distributed uniformly throughout the water column in low-resource areas.

Patterns in MOCNESS data. Resource heterogeneity in the water column was observed at 9 of the $30 \mathrm{MOC}$ NESS stations (max. fluorescence $>1$ ), and at 8 of these stations the distribution of the identified xlarge zooplankton (i.e. the predators: chaetognaths, jellyfish and the amphipod Themisto abyssorum) matched the distribution of the resource (Fig. 5). The distribution of the most abundant small zooplankter, the herbivore Calanus finmarchicus CIII, matched the resource at 3 stations and undermatched the resource at 6 of the 9 resource-heterogeneous stations (Fig. 5).

\section{DISCUSSION}

Habitat choice theory provides a powerful synthetic framework for dealing with the distribution of foraging organisms (Morris 2003). In pelagic communities, zooplankton distributions throughout the water column display patterns that can be explained by habitat choice. Extensive sampling of the above distributional patterns in marine systems is possible using automated sampling devices, as implemented in this study. The observed patterns of vertical distribution were repeated across transects (Fossheim 2006). When present together, different size classes of zooplankton partitioned the water column, sharply segregating in different depth habitats. Small zooplankton typically stayed below the food-rich layers occupied by large zooplankton.

The observed configuration of depth habitat use is consistent with the expectations of habitat choice theory for unequal prey competitors (Brown 1998). In
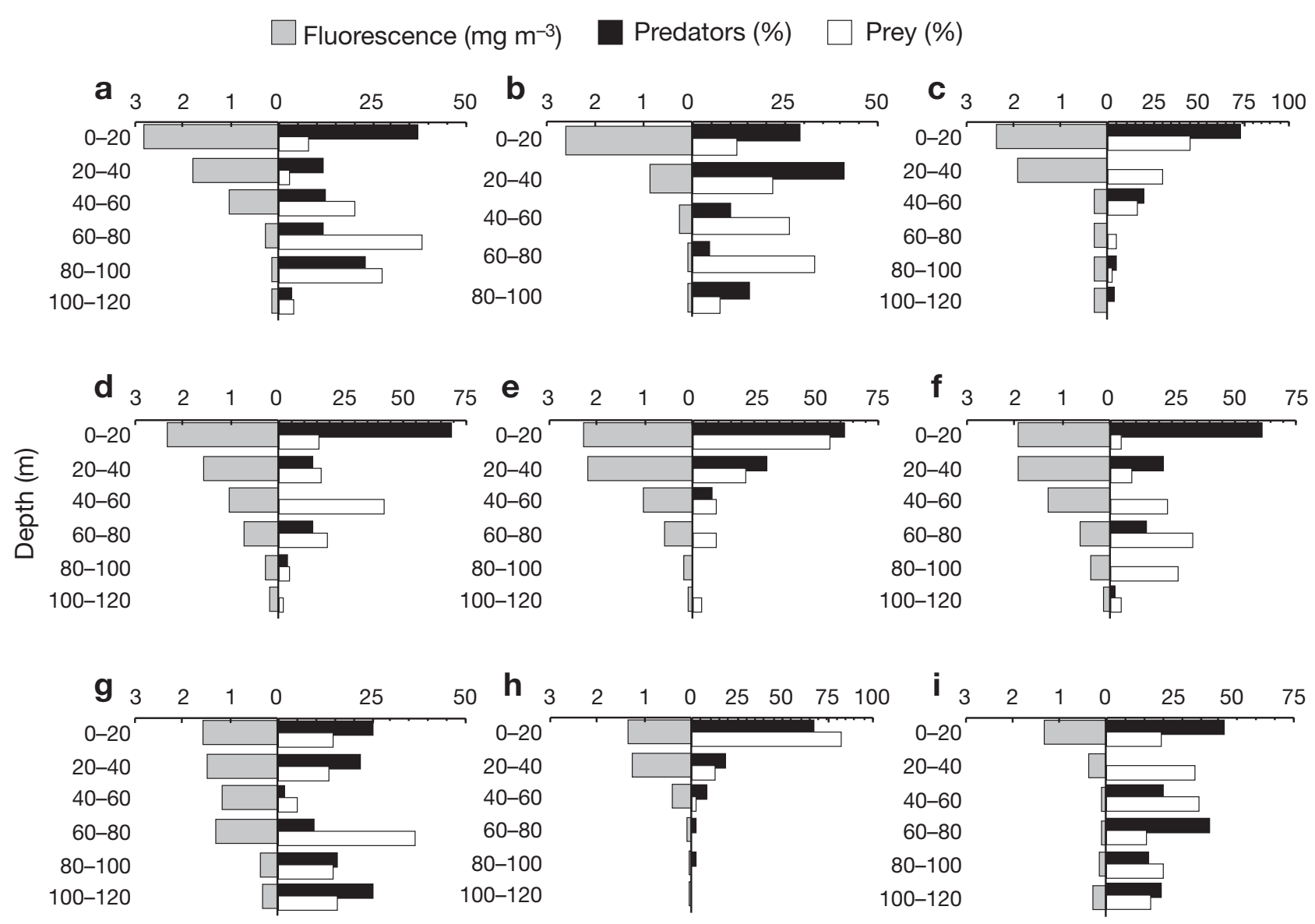

Fig. 5. Vertical distribution of fluorescence, extra large zooplankton (predators: chaetognaths, jellyfish and the amphipod Themisto abyssorum) and Calanus finmarchicus copepodite stage III (CIII) throughout heterogeneous water columns sampled with MOCNESS (see Fig. 1). Interpretation of resource matching by predators (xlarge zooplankton) and prey (C. finmarchicus CIII): (a) predator match, prey undermatch (b) predator match, prey undermatch (c) predator match, prey match; $(\mathrm{d})$ predator match, prey undermatch; (e) predator match, prey match; (f) predator match, prey undermatch; (g) predator no match, prey undermatch; (h) predator match, prey match; (i) predator match, prey undermatch 
the presence of size-selective predators with a preference for smaller prey, the small zooplankters are the competitively superior but more vulnerable prey. Small zooplankton therefore should choose the resource-poor habitat refuge, whereas larger zooplankton are competitively displaced from the habitat refuge and choose the resource-rich habitat with the predator (Fig. 6a). Medium-sized zooplankton positioned themselves at the interface between food-rich and foodpoor depth layers, where predation pressure was expected to decline rapidly and competition by small zooplankton was less intense. The observed depth habitat use by medium-sized zooplankton would thus balance starvation and predation risk, assuming an intermediate competitive ability and vulnerability relative to small and large zooplankton (Fossheim 2006).

The small zooplankton in our OPC data were mostly represented by Calanus finmarchicus CI through III, so our interpretation above implies that the smaller stages of $C$. finmarchicus press resources to a level lower than that tolerated by the larger C. finmarchicus stages, which are competitively displaced. The distribution of a
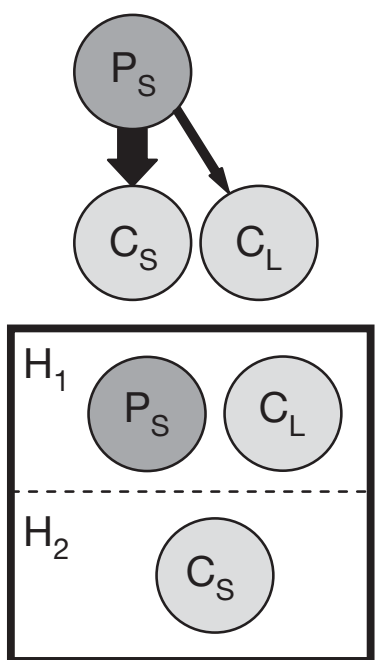

b
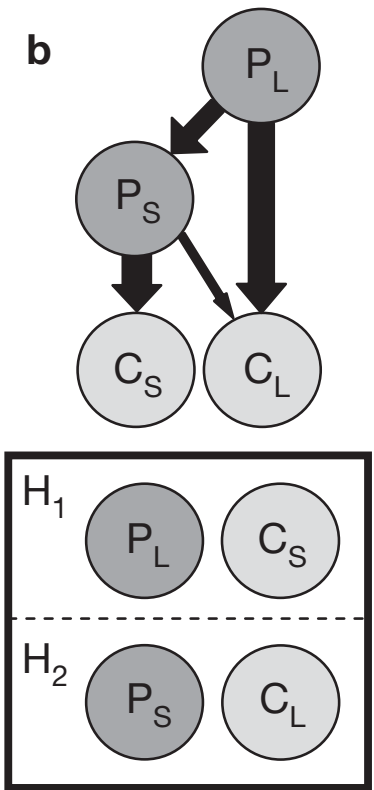

Fig. 6. Community modules (upper panels, arrow thickness depicts interaction strength) and corresponding adaptive configurations of habitat use by interacting foragers (lower panels). Resource availability is assumed to be heterogeneous among habitats $\left(\mathrm{H}_{1}\right.$, resource-rich habitat; $\mathrm{H}_{2}$, resource-poor habitat). (a) Configuration of habitat use for a 3-trophic level community module containing small zooplankton prey $\left(\mathrm{C}_{\mathrm{S}}\right.$ competitively superior but vulnerable to predation), large zooplankton prey $\left(\mathrm{C}_{\mathrm{L}}\right.$, competitively inferior and less vulnerable to predation), and their shared predator (adapted from Brown 1998). (b) Configuration of habitat use for a 4-trophic level community module containing zooplankton $\left(\mathrm{C}_{\mathrm{S}}\right.$ and $\left.\mathrm{C}_{\mathrm{L}}\right)$, invertebrate predators $\left(\mathrm{P}_{\mathrm{S}}\right)$ and planktivorous fish predators $\left(\mathrm{P}_{\mathrm{L}}\right)$ young copepodite stages of $C$. finmarchicus throughout the water column varies with latitude and season (Mauchline 1998). When the small copepodites are reported in surface waters, their distribution is often explained by optimal feeding conditions (Hirche et al. 2001). However, optimal feeding conditions alone cannot explain the deep distribution that we observed, since the small zooplankton are found in low resource areas (Fretwell \& Lucas 1969). However, this distribution can be explained by including predation risk from predators feeding selectively on small zooplankton (Lima 2002). When present, the xlarge zooplankton, which included predators of small copepodites, was found in the resource-rich habitats, as expected from multi-trophic level habitat choice theory (Sih 1998, Primicerio 2005), which holds that predators should hunt in the habitat nutritionally most profitable for their prey (Sih 1998).

The predators of small zooplankton identified in the MOCNESS samples (chaetognaths, jellyfish and the amphipod Themisto abyssorum) were matching the resource level of their prey at almost all resourceheterogeneous stations; the net samples are therefore consistent with multi-trophic level habitat choice theory and the OPC findings for the xlarge zooplankton. At the resource-heterogeneous net stations we also found undermatching of the resource by the most abundant small zooplankter, the herbivore Calanus finmarchicus CIII, further supporting our interpretation of the OPC data according to multi-trophic level habitat choice theory.

The MOCNESS data seem unsuitable to test habitat choice hypotheses that assume resource heterogeneity throughout the water column, since the MOCNESS samples covered a much smaller fluorescence range $(0$ to 2.8) than the OPC (0 to 6.6), missing the more resource-heterogeneous water columns. Segregation between small and large zooplankton was clear in the more heterogeneous columns (max. fluorescence $>3$ ) sampled by the OPC. This is a general sampling problem because high intensity patches (hot spots of fluorescence) are rare and therefore likely to be missed by low-coverage sampling equipment such as nets. Extensive sampling techniques (lacking taxonomic information), such as the OPC, are therefore necessary to test hypotheses on distribution of different zooplankton size groups in heterogeneous environments. Additionally, to overcome sampling challenges set by a strongly advective environment we might have to tolerate lower taxonomic resolution. Automated sampling techniques lacking taxonomic information can never replace net samples, but may provide complementary information, which is particularly valuable when sampling techniques are inter-calibrated, as was attempted in this study. 
The large zooplankton in our OPC data included Calanus finmarchicus CV. Late copepodite stages of C. finmarchicus are often reported in deep waters (Mauchline 1998), where their presence is explained by predator avoidance of size-selective predators that forage on the older, larger stages (Kiørboe 1997, Aksnes et al. 2004). In our study, size-selective predators of large zooplankton were probably absent. In the absence of their predators, the larger zooplankton aggregated near the surface, in the resource-rich habitat. It may also be speculated that large zooplankton were competitively displaced from the deeper water layers by smaller zooplankton (Mittelbach et al. 2004), which have lower resource demands (Mauchline 1998, Hirst \& Bunker 2003).

Along the Norwegian coast, size-selective predators of large zooplankton are typically represented by planktivorous fish (Huse \& Toresen 1996, Dale et al. 2001), which in the southern Barents Sea become more important in summer (Huse \& Toresen 1996). In the study area, newly hatched Mallotus villosus larvae, born in spring, feed on small zooplankton (Pedersen \& Fossheim 2008). In summer, these larvae will have reached juvenile stage and a size that allows them to feed on larger zooplankton. This ontogenetic niche shift changes the predation regime experienced by the different zooplankton sizes. Additionally, in the same period, larger fish larvae and juveniles (mainly Gadus morhua and Clupea harengus) are advected onto the shelf of the southern Barents Sea, contributing to the summer increase in predation risk for large zooplankton.

Assuming different predation regimes, habitat choice theory can explain the variation in distributions of ontogenetic stages of Calanus finmarchicus in late spring (this study) versus mid-summer (Aksnes et al. 2004). A mid-summer setting including planktivorous fish would thus involve a fourth trophic level where, according to theory, the fish predator is expected to reside in the resource-rich habitat (Rosenheim 2004). The fish predator will have a preference for larger zooplankton, including invertebrate predators (our xlarge size group) of small zooplankton. The small predators and the large zooplankton are then expected to seek the habitat refuge, whereas the small zooplankton will choose to stay in the resource-rich habitat with the fish predator (Fig. 6b). The fish predator protects small zooplankton from their main predators. This configuration of depth habitat use is found in zooplankton in mid-summer in Norwegian waters (Aksnes et al. 2004).

Our interpretation of the observed patterns of zooplankton distribution is different from the predatoravoidance hypothesis, which states that visual predators hunt near surface to be able to see their prey (sensory constraint) and that zooplankton prey move down to avoid visual predators (e.g. Aksnes et al. 2004). This explanatory framework, based on a sensory constraint of visual predators, misses the co-evolutionary aspect addressed by multi-trophic level habitat choice theory. The latter theory predicts that predators should hunt in the habitat most profitable for their prey independently of hunting strategy (visual, tactile or otherwise). As such, it represents a more general theory explicitly addressing the frequency-dependent nature of these behavioural adaptations. The multitrophic level habitat choice theory is able to explain the seasonal change in zooplankton configuration, whereas the visual predator-avoidance hypothesis cannot explain the spring configuration of zooplankton found in this study, since most of the identified predators are invertebrates (and non-visual predators) and thus not limited to the upper water column. We therefore recommend that theories based on frequencydependent behaviour (such as the multi-trophic level habitat choice theory), developed and extensively tested in terrestrial and limnological studies, be considered when interpreting small- and mesoscale patterns in the marine environment.

The present study shows that body size is an important component in predicting habitat choice by zooplankton consumers because it affects minimum maintenance levels and vulnerability to predators. Extensive fine-resolution data from an OPC allowed recent developments in habitat choice theory as applied to marine zooplankton to be tested. The observed configuration of habitat use is consistent with the expectations of multi-trophic level habitat choice theory with unequal prey competitors. Marine zooplankton seem to choose their habitat according to a balance between starvation and predation risk throughout the water column.

Acknowledgements. The authors thank A. Edvardsen for help with the integration of OPC size spectrum data and database extractions. We also thank the Captain and crew of RV 'Jan Mayen' and participants of the BAS 2 cruise in May 2001. The constructive comments of 3 anonymous reviewers greatly improved the quality of the manuscript. This work was financed by the Research Council of Norway through project no. $140290 / 140$.

\section{LITERATURE CITED}

Adler FR, Richards SA, de Roos AM (2001) Patterns of patch rejection in size-structured populations: beyond the ideal free distribution and size segregation. Evol Ecol Res 3: 805-827

Aksnes DL, Nejstgaard J, Sædberg E, Sørnes T (2004) Optical control of fish and zooplankton populations. Limnol Oceanogr 49:233-238

> Alonzo SH, Switzer PV, Mangel M (2003) Ecological games in space and time: the distribution and abundance of Antarctic krill and penguins. Ecology 84:1598-1607

Ashjian CJ, Campbell RG, Welch HE, Butler M, Van Keuren 
D (2003) Annual cycle in abundance, distribution, and size in relation to hydrography of important copepod species in the western Arctic Ocean. Deep-Sea Res I 50:1235-1261

Baier CT, Purcell JE (1997) Trophic interactions of chaetognaths, larval fish, and zooplankton in the South Atlantic Bight. Mar Ecol Prog Ser 146:43-53

Blachowiak-Samolyk K, Kwasniewski S, Richardson K, Dmoch K and others (2006) Arctic zooplankton do not perform diel vertical migration (DVM) during periods of midnight sun. Mar Ecol Prog Ser 308:101-116

Brown JS (1998) Game theory and habitat selection. In: Dugatkin LA, Reeve HK (eds) Game theory and animal behavior. Oxford University Press, Oxford, p 188-220

> Dale T, Kaartvedt S, Ellertsen B, Amundsen R (2001) Largescale oceanic distribution and population structure of Calanus finmarchicus in relation to physical food and predators. Mar Biol 139:561-574

Dalpadado P (2002) Inter-specific variations in distribution, abundance and possible life-cycle patterns of Themisto spp. (Amphipoda) in the Barents Sea. Polar Biol 25: 656-666

Davison AC, Hinkley DV (1997) Bootstrap methods and their application. Cambridge University Press, Cambridge

Dill LM, Heithaus MR, Walters CJ (2003) Behaviorally mediated indirect interactions in marine communities and their conservation implications. Ecology 84:1151-1157

Edvardsen A, Zhou M, Tande KS, Zhu YW (2002) Zooplankton population dynamics: measuring in situ growth and mortality rates using an Optical Plankton Counter. Mar Ecol Prog Ser 227:205-219

Folt CL, Burns CW (1999) Biological drivers of zooplankton patchiness. Trends Ecol Evol 14:300-305

Fossheim M (2006) Spatial structure of capelin larvae and zooplankton off the coast of northern Norway. PhD thesis, Department of Aquatic BioSciences, Norwegian College of Fishery Science, University of Tromsø

Fossheim M, Zhou M, Tande KS, Pedersen OP, Zhu Y, Edvardsen A (2005) Interactions between biological and environmental structures along the coast of northern Norway. Mar Ecol Prog Ser 300:147-158

Fossheim M, Tande KS, Semenova T, Timonin A (2006) Capelin larvae (Mallotus villosus) and zooplankton off the coast of northern Norway. J Plankton Res 28:585-595

Fretwell SD, Lucas HLJ (1969) On territorial behavior and other factors influencing habitat distribution in birds. I. Theoretical development. Acta Biotheor 19:16-36

Grabowski JH, Kimbro DL (2005) Predator-avoidance behavior extends trophic cascades to refuge habitats. Ecology 86:1312-1319

Heath MR, Dunn J, Fraser JG, Hay SJ, Madden H (1999) Field calibration of the Optical Plankton Counter with respect to Calanus finmarchicus. Fish Oceanogr 8:13-24

Herman AW (1992) Design and calibration of a new Optical Plankton Counter capable of sizing small zooplankton. Deep-Sea Res 39:395-415

Hirche HJ, Brey T, Niehoff B (2001) A high-frequency time series at Ocean Weather Ship Station M (Norwegian Sea): population dynamics of Calanus finmarchicus. Mar Ecol Prog Ser 219:205-219

Editorial responsibility: Matthias Seaman, Oldendorf/Luhe, Germany
Hirst AG, Bunker AJ (2003) Growth of marine planktonic copepods: global rates and patterns in relation to chlorophyll a, temperature, and body weight. Limnol Oceanogr 48:1988-2010

Huse G, Toresen R (1996) A comparative study of the feeding habits of herring (Clupea harengus, Clupeidae, L) and Capelin (Mallotus villosus, Osmeridae, Muller) in the Barents Sea. Sarsia 81:143-153

Kiørboe T (1997) Population regulation and role of mesozooplankton in shaping marine pelagic food webs. Hydrobiologia 363:13-27

Leibold MA, Tessier AJ (1997) Habitat partitioning by zooplankton and the structure of lake ecosystems. In: Streit B, Städler T, Lively CM (eds) Evolutionary ecology of freshwater animals. Birkhäuser Verlag, Basel, p 3-30

Lima SL (2002) Putting predators back into behavioral predator-prey interactions. Trends Ecol Evol 17:70-75

Mauchline J (1998) The biology of calanoid copepods. Academic Press, New York

> Mittelbach GG, Darcy Hall TL, Dorn NJ, Garcia EA, Steiner CF, Wojdak JM (2004) The impact of density-independent mortality on species coexistence: an experimental test with zooplankton. Oikos 107:415-421

Morris DW (2003) Toward an ecological synthesis: a case for habitat selection. Oecologia 136:1-13

> Pedersen T, Fossheim M (2008) Diet of 0-group stages of capelin (Mallotus villosus), herring (Clupea harengus) and cod (Gadus morhua) during spring and summer in the Barents Sea. Mar Biol 153:1037-1046

> Persson L, Andersson J, Wahlstrom E, Eklov P (1996) Sizespecific interactions in lake systems: predator gape limitation and prey growth rate and mortality. Ecology 77 : 900-911

Pinel-Alloul B (1995) Spatial heterogeneity as a multiscale characteristic of zooplankton community. Hydrobiologia 300-301:17-42

Primicerio R (2005) Predator mediated coexistence in zooplankton: the importance of behavioural mechanisms. Verh Int Verein Limnol 29:158-162

> Rosenheim JA (2004) Top predators constrain the habitat selection games played by intermediate predators and their prey. Isr J Zool 50:129-138

Sih A (1998) Game theory and predator-prey response races. In: Dugatkin LA, Reeve HK (eds) Game theory and animal behavior. Oxford University Press, Oxford, p 221-238

Tokeshi M (1999) Species coexistence-ecological and evolutionary perspectives. Blackwell Science, Oxford

Tönnesson K, Tiselius P (2005) Diet of the chaetognaths Sagitta setosa and $S$. elegans in relation to prey abundance and vertical distribution. Mar Ecol Prog Ser 289: $177-190$

> Woodward G, Ebenman B, Ernmerson M, Montoya JM, Olesen JM, Valido A, Warren PH (2005) Body size in ecological networks. Trends Ecol Evol 20:402-409

> Zhang X, Roman M, Sanford A, Adolf H, Lascara C, Burgett R (2000) Can an optical plankton counter produce reasonable estimates of zooplankton abundance and biovolume in water with high detritus? J Plankton Res 22: $137-150$

Submitted: August 14, 2007; Accepted: March 13, 2008 Proofs received from author(s): July 4, 2008 